\title{
Quantum Dashes and Quantum Rods: Optical Properties and Application Prospects
}

\author{
G. SEKK \\ Institute of Physics, Wrocław University of Technology, Wybrzeże Wyspiańskiego 27, 50-370 Wrocław, Poland \\ There are reported the optical properties of novel quasi-zero-dimensional semiconductor structures obtained \\ by self-assembly during the molecular beam epitaxy process. The studies has been focused on strongly asymmetric \\ systems, called quantum dashes, quantum rods or columnar quantum dashes, and their potential applications \\ in light sources or amplifiers for telecommunication and data transmission or single photon sources and other \\ quantum electrodynamics based emitters. There is discussed the linear polarization degree of the edge emission \\ of the columnar structures with respect to their potential for a polarization independent semiconductor optical \\ amplifier at $1.55 \mu \mathrm{m}$. Additionally, there have been analyzed the photoluminescence spectra of single quantum \\ dashes and single quantum rods in order to detect the exciton/biexciton emission, determine the biexciton binding \\ energy and predict the exciton to biexciton radiative lifetimes ratio basing on a few level rate equation model.
}

PACS numbers:

\section{Introduction}

The increasing requirements of present optoelectronics (including the telecommunication and data transmission market), together with the appearing new concepts for the realization of secure information transfer or quantum computing, are the main driving forces of the development in the nanostructure technologies, including epitaxial growth of self-assembled quasi-zero-dimensional systems. Hence, there have appeared new kinds of quantum-dot-like objects where their shape engineering is the key issue to tailor the electronic or optical properties. For instance, it was possible to grow strongly in-plane elongated dots or finite size quantum wires (the so-called quantum dashes, QDashes), which have been demonstrated as very efficient light emitters in the near infrared and found their application as the active region of laser diodes for telecommunication [1]. On the other hand, the fabrication of high aspect ratio quantum dots (called columnar quantum dots or quantum rods) has allowed achieving strongly enhanced emission intensity from the structure edge in the TM (transverse magnetic) linear polarization (with respect to the transverse electric, TE), which gives a potential for the realization of fully polarization independent quantum-dot-like semiconductor optical amplifier [2-5].

In this work, we discuss the optical properties of quantum-dot-like nanoobjects, whose shape has been intentionally made strongly asymmetric in order to control certain of their electronic structure features. Quantum dashes with significantly increased height (columnar quantum dashes, CQDashes) are investigated from the viewpoint of their polarization properties and potential for polarization independent emission (and further the gain function of the device) in the range of $1.55 \mu \mathrm{m}$. The quantum dashes and quantum rods, on the other hand, are investigated by microphotoluminescence $(\mu \mathrm{PL})$ in order to detect the single exciton $(\mathrm{X})$ and biexciton $(\mathrm{XX})$ emission and determine the biexciton binding energy in these uncommon geometry "dots". Additionally, the dynamics of the exciton/biexciton cascade, based on a few level rate equation model, could be estimated and predictions on internal exciton kinetics are given.

\section{Experimental}

The characteristic geometry features of the investigated structures are schematically shown in Fig. 1. All of them can be grown by molecular beam epitaxy and their growth parameters have been presented elsewhere [1, 6-9]. The structural characterization revealed that the InAs quantum dashes grown on InP substrate are usually more than $100 \mathrm{~nm}$ long, whereas their dimensions in the cross-section (perpendicular to the largest size), shape of which can be well modeled by a triangle, are directly correlated (and hence well controlled) with the amount of the deposited InAs material [10] and of the order of $10-30 \mathrm{~nm}$ in base width and several $\mathrm{nm}$ in height. The InGaAs/GaAs quantum rods (QRs), whose shape is parallelepiped with a rhombus base, have the characteristic vertical to lateral sizes ratio of 40 to $10 \mathrm{~nm}$ (i.e. the aspect ratio of approximately 4 in contrast to about 0.2 for common self-assembled $\operatorname{In}(\mathrm{Ga})$ As quantum dots). The columnar InGaAs quantum dashes on InP used for this study have the lengths similar as for the usual dashes, the width of about 10-15 nm, and height varied by the number of deposited InAs/GaAs bilayers, in the range of a few nm up to even more than $20 \mathrm{~nm}$. There are two other 
details of the QRs and CQDashes which are worth noting and have important implications for their electronic and optical properties. The growth mode (close stacking or deposition of a short period superlattice with sometimes submonolayer unit thicknesses) and the atoms interdiffusion combined with indium segregation process cause, first of all, the formation of an "immersion layer" in the lateral direction (i.e. a material filling the areas between the rods/dashes (see Fig. 1) and differing in the composition) and functioning as a confinement potential barrier in plane. Second, the compositions over the $\mathrm{rod} / \mathrm{dash}$ and across the immersion layer are fairly uniform $[6,7]$. (a)

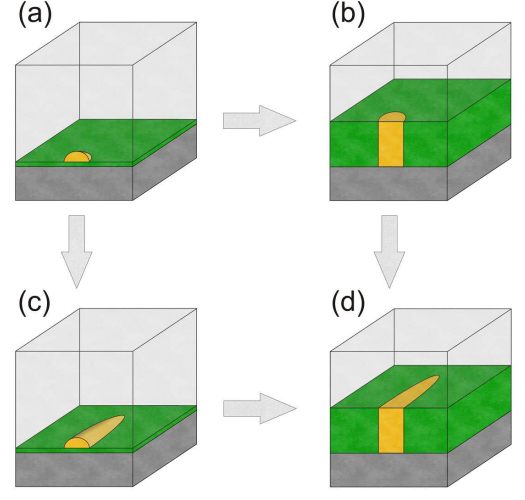

Fig. 1. Schemate of the geometry variations from typical self-assembled quantum dots (a), to quantum rods (b), quantum dashes (c) and columnar quantum dashes $(\mathrm{d})$. The green areas mean wetting layer in the quantum dot and quantum dash structures and immersion layer in quantum rod and columnar quantum dash structures.

All the measurements have been performed using a microphotoluminescence setup based on a long working distance $(20 \mathrm{~mm})$ and high numerical aperture $(0.4) \mathrm{mi}-$ croscope objective assuring the diffraction limited spatial resolution of approximately $2 \mu \mathrm{m}$, depending on the used excitation wavelength. It has been combined with a single grating $0.55 \mathrm{~m}$ focal length monochromator equipped with a liquid nitrogen cooled multichannel linear InGaAs charged coupled device and a thermoelectrically cooled long wavelength InGaAs PIN photodiode serving as detectors. Further experimental details have been described elsewhere [11-13].

\section{Results and discussion}

\subsection{Polarization properties of columnar quantum dash structures}

As the InAs quantum dashes on InP substrate with lattice matched (to $\mathrm{InP}$ ) quaternary $\mathrm{In}_{0.53} \mathrm{Ga}_{0.23} \mathrm{Al}_{0.24} \mathrm{As}$ barriers have been proven to be easily spectrally tunable over $1.55 \mu \mathrm{m}[1,14,15]$, creating columnar (tall) dashes has been considered as a way of obtaining a polarization independence of the edge emission in that range for the optical amplifier application. The concept bases on affecting the linear polarization dependent selection rules for the optical transition and for the light propagation vector in the structure plane (perpendicularly to the growth direction). Increasing the object height (the aspect ratio) weakens the quantum confinement in that direction and leads to the increased light hole contribution to the mixed valence band states causing the intensity enhancement in the TM polarization (along the growth direction) $[2-5,9,16]$. An additional effect is the composition contrast between the column and the in-plane surrounding (immersion layer) which modifies the strain distribution and hence the heavy hole-light hole bands mixing of the valence states and also the TM/TE intensities ratio.

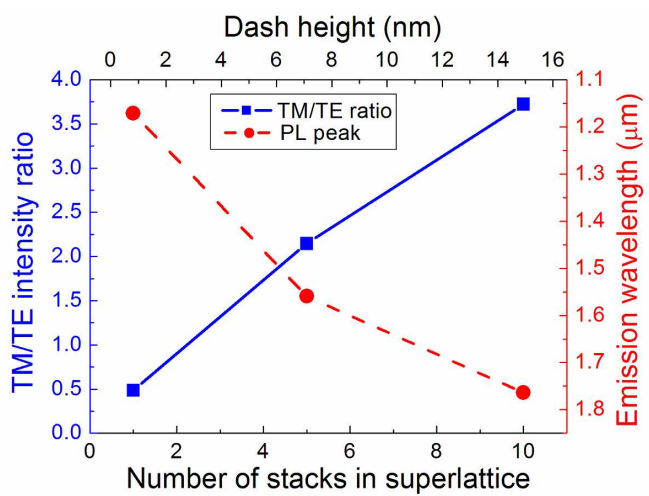

Fig. 2. TM to TE polarized PL intensity ratio (blue curve) and the dash-related emission wavelength (red) as a function of a number of stacked InAs/GaAs bilayers (column height) in the columnar dash structures.

In Fig. 2 is shown a dependence of this ratio on the number of InAs/GaAs bilayers in the close stacking columnar dash growth (taken as an integrated CQDash-related photoluminescence (PL) intensity from the structure edge cleaved perpendicularly to the largest dash size and measured for both the linear polarizations). As a reference, the estimated corresponding column height is indicated in the upper axis. The TM/TE intensities ratio increase almost linearly with the number of stacked bilayers and for this nominal thicknesses combination (0.7 $\mathrm{nm} \mathrm{InAs} / 0.8 \mathrm{~nm}$ GaAs) reaches 1 (the polarization insensitive case) for about 3 pairs. The left axis shows how the room temperature PL peak wavelength changes with the number of stacks (dash height). The wavelength of interest is reached for approximately $5 \mathrm{InAs} / \mathrm{GaAs}$ pairs grown, which is slightly mismatched with the optimum polarization properties, i.e. further design and growth optimization is necessary.

Figure 3 shows a room temperature PL spectrum for an 8 times stacked columnar dash structure with an InAs to GaAs layer thicknesses of about 0.8 and $0.7 \mathrm{~nm}$, respectively, chosen in a way to have the average material composition of $\operatorname{In}_{0.53} \mathrm{Ga}_{0.47}$ As (which as a uniform layer would be lattice matched to the substrate). The spectrum exhibits actually two emission bands at 1.7 and 


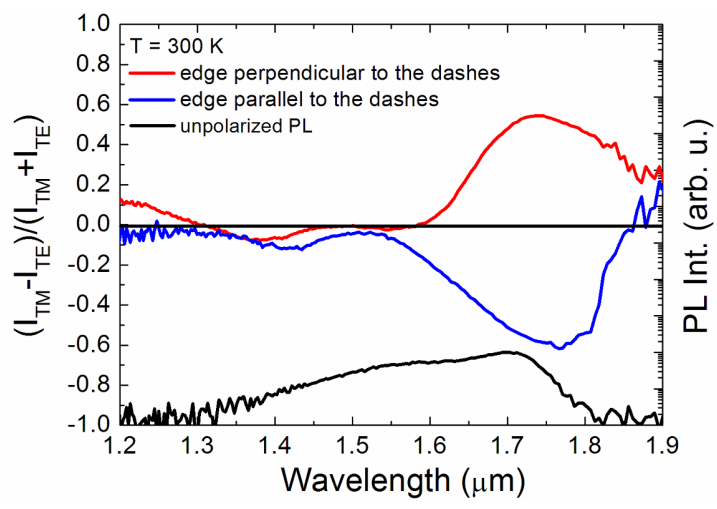

Fig. 3. Unpolarized photoluminescence from an InGaAs/InP columnar dash structure (black). Polarization degree functions for two perpendicular sample edges (red and blue).

$1.55 \mu \mathrm{m}$. Previous studies based on a comparison to modulated reflectivity spectra (absorption-like) and energy level calculations $[9,17]$ have revealed that the two bands correspond to the emission from the dashes and the immersion layer, respectively. In Fig. 3, there is presented the polarization degree (PD) function for both the sample edges (parallel and perpendicular to the largest dash size) defined as:

$$
\mathrm{PD}=\frac{I_{\mathrm{TM}}-I_{\mathrm{TE}}}{I_{\mathrm{TM}}+I_{\mathrm{TE}}}
$$

where $I_{\mathrm{TE}(\mathrm{TM})}$ is the PL intensity for the TE (TM) polarization. These two functions show that the CQDash related PL (at $1.7 \mu \mathrm{m})$ is strongly polarized: TE, i.e. in plane, for the edge parallel to the dashes (driven by the dash length dominating the cross-section geometry) as expected for the "flat" structure shape; TM for the edge perpendicular to the dashes showing predominance of the column height in this cross-section. The most interesting is the PD at $1.55 \mu \mathrm{m}$ which is almost zero for both the edges. The latter shows that the immersion layer can be the polarization independent emitter at the proper wavelength for this InAs/GaAs bilayer combination (and remaining growth parameters like the deposition rate or temperature, which affect the compositions by the segregation and interdiffusion effects). The explanation for this behavior is the following. The immersion layer can be treated as a relatively thick $(\approx 10 \mathrm{~nm})$ quantum well, which is nominally slightly tensily strained, because the segregation effect will cause the CQDashes being In-rich (with respect to the average content of 0.53), where the surrounding immersion layer becomes In-poor. This pushes the bulk-like light hole band deeper into the well, or in other words, increases the light hole character of the valence band states. It is additionally combined with the strongly modified strain within the layer caused by the existence of the compressively strained dashes (the strain function in the layer plane is a function strongly oscillating between the negative and positive values). In total, the valence band becomes mixed to that degree which gives equal TE and TM polarizations at exactly $1.55 \mu \mathrm{m}$ at room temperature.

\subsection{Photoluminescence from a single InAs/InP quantum dash}

Due to the reasons mentioned above (spectral range related), but also some others like the expected enhanced oscillator strength (large dashes size compared to the dots increases the exciton coherence volume) or distinct and well defined linear polarization properties, the quantum dashes can be interesting as the light emitters in applications using the quantum electrodynamics experiments in the regime of a single dash (single exciton) and single photon especially, like for instance single photon sources or micro (nano) lasers. For these the single dash emission properties (including the biexciton) are necessary to be known. However, the single dash studies are not trivial (hence almost no reports are available $[12,18]$ ) because of a naturally very high surface dash density (the surface coverage can reach $50 \%$, which is by almost two orders of magnitude larger than in typical quantum dot structures) and the spectral range in which the available detectors efficiency (detectivity and noise) are worse than in the $1 \mu \mathrm{m}$ range operated by silicon based photosensors.

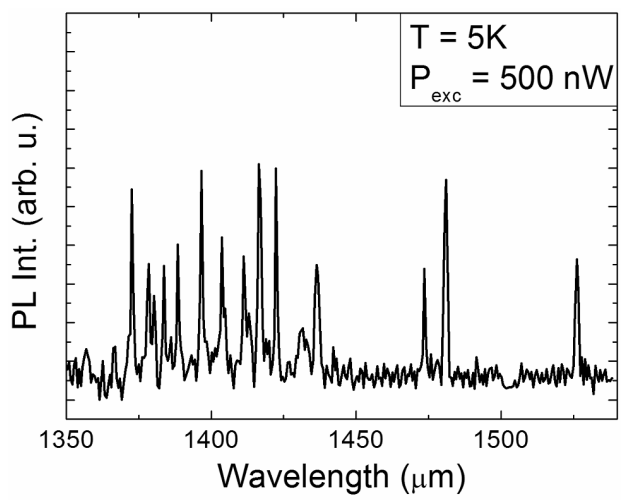

Fig. 4. Low temperature photoluminescence spectrum for a $275 \times 550 \mathrm{~nm}$ mesa with InAs/InP quantum dashes. The excitation power $P_{\text {exc }}$ is measured outside the cryostat.

Figure 4 shows a low temperature PL spectrum from a quantum dash structure on which small mesas have been patterned in order to additionally (beside the small probing area in the $\mu \mathrm{PL}$ experiment) limit the number of investigated objects. It concerns a mesa of $275 \times 500 \mathrm{~nm}$ size (elongated along the dashes) with about 20 dashes inside. The spectrum reveals well separated sharp lines corresponding to the emission from different dashes. The broad emission range (related to the large dashes nonuniformity) allows observing the individual lines on one hand, but on the other is responsible for covering quite a broad spectral range from above $1.3 \mathrm{~m}$ to almost $1.55 \mu \mathrm{m}$ in one sample.

Figure 5 shows the excitation power dependence of a single quantum dash PL measured with the enhanced 


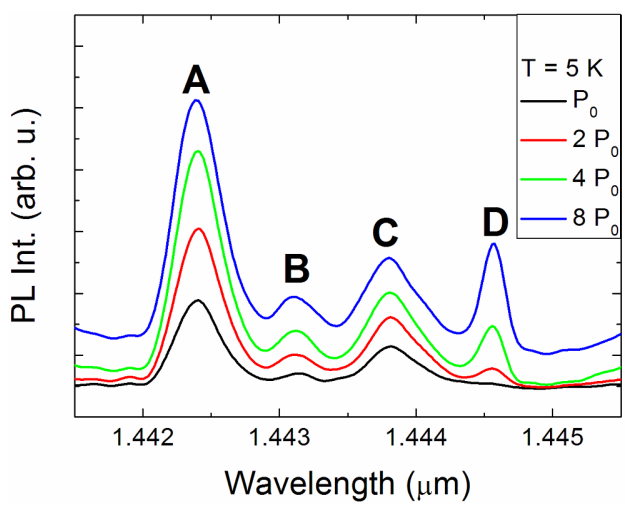

Fig. 5. Low temperature $\mu \mathrm{PL}$ spectra revealing single dash emission lines, taken on a $275 \times 550 \mathrm{~nm}$ mesa for several excitation powers $\left(P_{0}=250 \mathrm{nW}\right.$ measured outside the cryostat).

spectral resolution (below $100 \mu \mathrm{eV}$ ). There can be observed four lines (labeled A, B, C and D). The intensity of the first three of them goes up proportionally to the excitation power increase (and then starts saturating) whereas the last one (D) appears at higher excitation but then increases superlinearly and hence can be suspected to be related to a biexciton emission.

In order to explain the observed behavior and confirm the lines origin (relate the biexciton to an exciton line from the same dash and determine the biexciton binding energy) the excitation power dependence of the emission intensity has been modeled by using a few level rate equations expressed in a general form as

$$
\frac{\mathrm{d} p_{i}}{\mathrm{~d} t}=\frac{p_{i+1}}{\tau_{i+1}}+p_{i-1} g-p_{i}\left(\frac{1}{\tau_{i}}+g\right),
$$

where $p_{i}$ is the probability of a system occupation with one electron-hole pair (exciton), $\tau_{i}$ is the respective recombination time $\left(1 / \tau_{i}\right.$ is the recombination rate) and $g$ is the generation rate (proportional to the excitation power). For $i=0$ there is no exciton in the system (corresponds to its ground state). If we limit the consideration to only three level system, schematically shown in Fig. 6 (where the first excited state is one exciton, and second is a biexciton), this equation for one electron-hole pair $(i=1$, which we replace with $i=X)$ transforms into

$$
\frac{\mathrm{d} p_{X}}{\mathrm{~d} t}=-\frac{p_{X}}{\tau_{X}}+\frac{p_{X X}}{\tau_{\mathrm{XX}}}+g p_{0}-g p_{X}
$$

and for two excitons (biexciton) it takes the form

$$
\frac{\mathrm{d} p_{X X}}{\mathrm{~d} t}=-\frac{p_{X X}}{\tau_{X X}}+g p_{X},
$$

where the direct creation of biexcitons is neglected.

Neglecting the possible contribution (occupation probability) of the higher levels and creation of charged excitons allows writing

$$
p_{0}+p_{X}+p_{X X}=1
$$

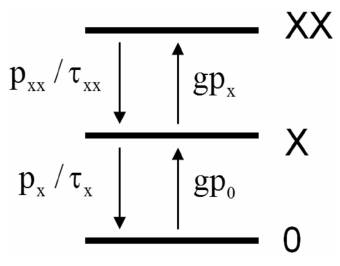

Fig. 6. Schematic representation of the three level exciton system.

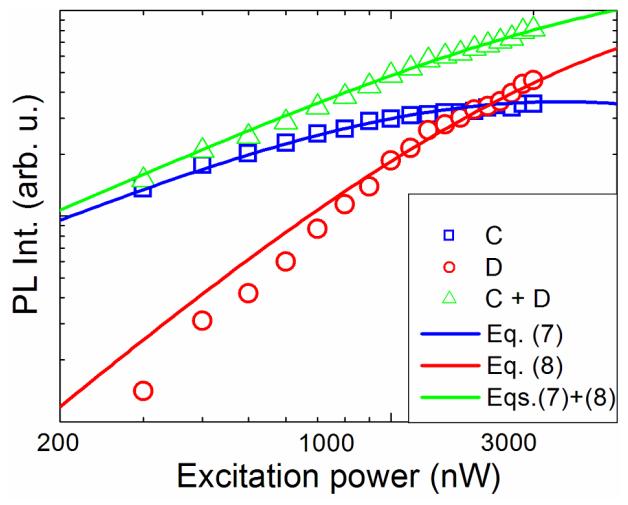

Fig. 7. Excitation power dependence of the $\mathrm{C}$ and $\mathrm{D}$ PL lines from Fig. 5. Symbols mean the experimental data, whereas the solid lines correspond to the theoretical curves after Eqs. (7) and (8) for $\tau_{X} / \tau_{X X}=2$.

$$
\frac{\mathrm{d} p_{X}}{\mathrm{~d} t}=-\frac{p_{X}}{\tau_{X}}+\frac{p_{X X}}{\tau_{\mathrm{XX}}}+g\left(1-2 p_{X}-p_{X X}\right) .
$$

In a stationary case $\left(\frac{\mathrm{d} p_{X}}{\mathrm{~d} t}=0\right.$ and $\left.\frac{d p_{X X}}{d t}=0\right)$ and taking into account that the respective emission intensity is $I_{X(X X)} \approx \frac{p_{X(X X)}}{\tau_{X(X X)}}$ the exciton and biexciton emission intensity dependence on the generation rate (excitation power) can be expressed as

$$
I_{X} \propto \frac{g}{1+g \tau_{X}+g^{2} \tau_{X} \tau_{X X}}
$$

and

$$
I_{X X} \propto \frac{g^{2} \tau_{X}}{1+g \tau_{X}+g^{2} \tau_{X} \tau_{X X}}
$$

The latter two equations (in the range of low generation rates) reduce to the linear and parabolic dependences, respectively. They are dependent on only one parameter which is exciton to biexciton lifetimes ratio $\tau_{X} / \tau_{\mathrm{XX}}$, which is typically assumed to be 2 (for the systems where the internal exciton relaxation from the bright to the dark states related with the spin flip is slow [19]). Figure 7 shows the dependence of the $\mathrm{C}$ and $\mathrm{D}$ lines intensity on the excitation power fitted by the theoretical relations (7) and (8). The obtained agreement (the model could not fit line $\mathrm{D}$ with any of the other observed lines, i.e. A and $\mathrm{B}$ ) confirms that $\mathrm{C}$ and $\mathrm{D}$ correspond to the exciton and biexciton emission from the same quantum dash. It is additionally supported by the agreement for the sum of the $X$ and $X X$ intensities (green points and curve in Fig. 7). This allows determining unambiguously the and hence 
biexciton binding energy to be $0.4 \mathrm{meV}$ for these dashes. Similar values could be obtained for other detected $X X$ cases.

\subsection{Emission from a single InGaAs/GaAs quantum rod}

Similar motivation as in the previous section concerns the quantum rods, which however, as based on GaAs substrate, can potentially be interesting for the applications at shorter wavelengths (up to $1.3 \mu \mathrm{m}$ usually). The surface density in this case is much lower than for the dashes $\left(10^{10} \mathrm{~cm}^{-2}\right)$ so in order to select the single emission lines the mesa structures patterning was not necessary [13]. Figure 8 shows the excitation dependent low temperature PL spectra exhibiting two emission lines. The intensity of the one at longer wavelength changes faster and therefore has been assigned as $X X$.

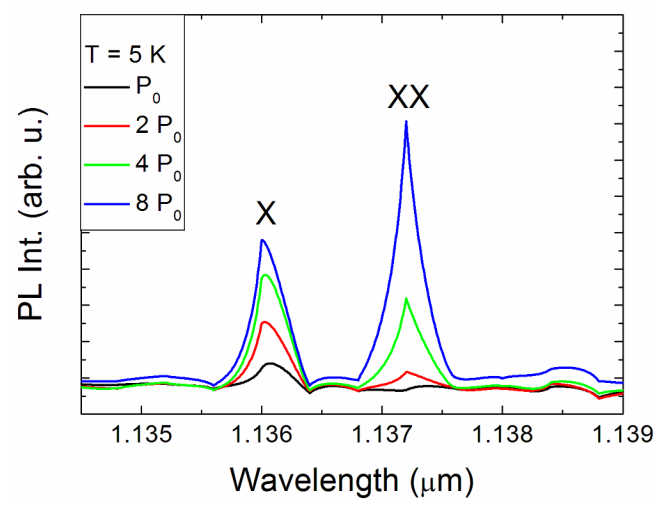

Fig. 8. Low temperature $\mu \mathrm{PL}$ spectra revealing single quantum rod emission lines for several excitation powers $\left(P_{0}=200 \mathrm{nW}\right.$ measured outside the cryostat).

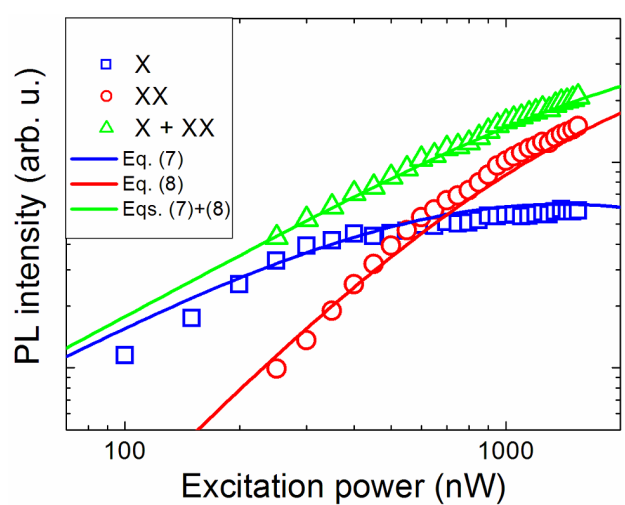

Fig. 9. Excitation power dependence of the $X$ and $X X$ lines from Fig. 8. Symbols mean the experimental data, whereas the solid lines correspond to the theoretical curves after Eqs. (7) and (8) for $\tau_{X} / \tau_{X X}=4$.

The integrated intensities for both observed lines are plotted as a function of the excitation power in Fig. 9. In this case the same model as in Sect. 3.2 has been used, but the satisfactory agreement could be obtained for $\tau_{X} / \tau_{X X}=4$, which based on Ref. [18] is an indication of a fast spin flip relaxation rate between the bright and dark states of an exciton in quantum rods. It has found a confirmation for some other exciton/biexciton cases in the investigated QRs structure, in spite of quite a broad distribution of the $X X$ binding energies, ranging from 1 to almost $3 \mathrm{meV}$.

\section{Conclusion}

There have been investigated the selected optical properties of epitaxially grown strongly asymmetric nanostructures. Based on the linear polarization resolved photoluminescence spectra from a cleaved edge of the samples it has been shown that the so called columnar quantum dashes obtained in the InGaAs/InP material system are very perspective for the polarization insensitive gain medium in semiconductor optical amplifiers at $1.55 \mu \mathrm{m}$. Moreover, the single dot spectroscopy allowed detecting the emission related to an exciton and biexciton from single quantum dashes and single quantum rods, and using the rate equation model determining the biexciton binding energy and exciton to biexciton radiative lifetimes ratio for both kinds of the systems.

\section{Acknowledgments}

The author would like to thank Jan Misiewicz from Institute of Physics, Wrocław University of Technology, for helpful comments and suggestions and for critical reading the manuscript, Paweł Podemski and Anna Musiał from the same Institute for the assistance in the spectroscopy related part of the work. Sven Höfling, Sebastian Hein and Alfred Forchel from Applied Physics Department, University of Würzburg, Andrea Fiore from Department of Applied Physics, Eindhoven University of Technology, and Lianhe Li from University of Leeds are acknowledged for the samples fabrication and the discussion of results.

\section{References}

[1] J.P. Reithmaier, G. Eisenstein, A. Forchel, Proc. IEEE 95, 1779 (2007) and references therein.

[2] T. Kita, N. Tamura, O. Wada, M. Sugawara, Y. Nakata, H. Ebe, Y. Arakawa, Appl. Phys. Lett. 88, 211106 (2006).

[3] K. Kawaguchi, N. Yasuoka, M. Ekawa, H. Ebe, T. Akiyama, M. Sugawara, Y. Arakawa, Jap. J. Appl. Phys. 47, 2888 (2008).

[4] K. Kawaguchi, N. Yasuoka, M. Ekawa, H. Ebe, T. Akiyama, M. Sugawara, Y. Arakawa, Appl. Phys. Lett. 93, 121908 (2008).

[5] P. Ridha, L. Li, A. Fiore, G. Patriarche, M. Mexis, P. Smowton, Appl. Phys. Lett. 91, 191123 (2007).

[6] L.H. Li, G. Patriarche, M. Rossetti, A. Fiore, J. Appl. Phys. 102, 033502 (2007)

[7] L.H. Li, G. Patriarche, P. Ridha, M. Rossetti, J. Andrzejewski, G. Sęk, J. Misiewicz, A. Fiore, IEEE J. Sel. Top. Quantum Electron. 14, 1204 (2008). 
[8] S. Hein, P. Podemski, G. Sęk, J. Misiewicz, P. Ridha, A. Fiore, G. Patriarche, S. Höfling, A. Forchel, Appl. Phys. Lett. 94, 241113 (2009).

[9] P. Podemski, G. Sęk, K. Ryczko, J. Misiewicz, S. Hein, S. Höfling, A. Forchel, G. Patriarche, Appl. Phys. Lett. 93, 171910 (2008).

[10] A. Sauerwald, T. Kümmell, G. Bacher, A. Somers, R. Schwertberger, J.P. Reithmaier, A. Forchel, Appl. Phys. Lett. 86, 253112 (2005).

[11] G. Sęk, P. Podemski, W. Rudno-Rudzinski, Z. Gumienny, J. Misiewicz, Opt. Appl. 37, 439 (2007).

[12] G. Sek, P. Podemski, A. Musiał, J. Misiewicz, S. Hein, S. Höfling, A. Forchel, J. Appl. Phys. 105, 086104 (2009).

[13] G. Sęk, P. Podemski, J. Misiewicz, L.H. Li, A. Fiore, G. Patriarche, Appl. Phys. Lett. 92, 021901 (2008).

[14] W. Rudno-Rudziński, R. Kudrawiec, P. Podemski, G. Sęk, J. Misiewicz, A. Somers, R. Schwertberger, J.P. Reithmaier, A. Forchel, Appl. Phys. Lett. $\mathbf{8 9}$ 031908 (2006).
[15] G. Sęk, P. Podemski, R. Kudrawiec, J. Misiewicz, A. Somers, S. Hein, S. Höfling, J.P. Reithmaier, A. Forchel, Proc. SPIE 6481, 64810F (2007).

[16] T. Saito, H. Ebe, Y. Arakawa, T. Kakitsuka, M. Sugawara, Phys. Rev. B 77, 195318 (2008)

[17] G. Sęk, P. Podemski, J. Andrzejewski, J. Misiewicz, S. Hein, S. Höfling, A. Forchel, Appl. Phys. Expr. 2, 061102 (2009).

[18] T. Mensing, L. Worschech, R. Schwertberger, J. Reithmaier, A. Forchel, Appl. Phys. Lett. 82, 2799 (2003).

[19] G.A. Narvaez, G. Bester, A. Franceschetti, A. Zunger, Phys. Rev. B 74, 205422 (2006). 